\title{
Causality constraints in large $N$ QCD coupled to gravity
}

\author{
Jared Kaplan and Sandipan Kundu๑* \\ Department of Physics and Astronomy, Johns Hopkins University, 3400 N, \\ Charles Street, Baltimore MD 21218, USA
}

(Received 27 October 2020; accepted 27 July 2021; published 2 September 2021)

\begin{abstract}
Confining gauge theories contain glueballs and mesons with arbitrary spin, and these particles become metastable at large $N$. However, metastable higher-spin particles, when coupled to gravity, are in conflict with causality. This tension can be avoided only if the gravitational interaction is accompanied by interactions involving other higher-spin states well below the Planck scale $M_{\mathrm{pl}}$. These higher-spin states can come from either the QCD sector or the gravity sector, but both these resolutions have some surprising implications. For example, QCD states can resolve the problem since there is a nontrivial mixing between the QCD sector and the gravity sector, requiring all particles to interact with glueballs at tree-level. If gravity sector states restore causality, any weakly coupled UV completion of the gravity sector must have many stringy features, with an upper bound on the string scale $M_{\text {string }} \lesssim \sqrt{M_{\mathrm{pl}} \Lambda_{\mathrm{QCD}} / N}$, where $\Lambda_{\mathrm{QCD}}$ is the confinement scale.
\end{abstract}

DOI: 10.1103/PhysRevD.104.L061901

\section{INTRODUCTION}

In the early days of quantum chromodynamics (QCD), 't Hooft pointed out that there is an unconventional systematic expansion obtained by taking the number of colors $N \rightarrow \infty$ and the gauge coupling $g \rightarrow 0$ with 't Hooft coupling $\lambda=g^{2} N=$ fixed [1,2]. Any such confining gauge theory is characterized by a confinement scale $\Lambda_{\mathrm{QCD}}$ where the 't Hooft coupling becomes strong. This scale determines the characteristic mass and physical size of generic hadrons bound together by the confining force.

It has been long believed that the confinement persists even at large $N$. This assumption leads to a classic result about the scaling of the correlators or scattering amplitudes of mesons $(\pi)$ and glueballs $(G)$ in the large $N$ limit (in the absence of gravity) [1-3]

$$
\left\langle G_{1} \cdots G_{n} \pi_{1} \cdots \pi_{p}\right\rangle \sim \frac{1}{N^{n+\frac{p}{2}-1-\delta_{p, 0}}},
$$

where amplitudes for free propagation are normalized so that they are independent of $N$. Therefore, the lifetime of a meson is rather long $\sim O(N)$. Glueballs are even more stable with typical lifetime of order $O\left(N^{2}\right)$. Furthermore, it is expected that QCD contains glueballs and mesons of spin $J>2$. On physical grounds, this is obvious since it is

\footnotetext{
*kundu@jhu.edu
}

Published by the American Physical Society under the terms of the Creative Commons Attribution 4.0 International license. Further distribution of this work must maintain attribution to the author(s) and the published article's title, journal citation, and DOI. Funded by SCOAP . possible to construct color singlet states with arbitrary spin by spinning quarks and gluons. The scaling relation (1) implies that in the exact $N=\infty$ limit these higher-spin mesons and glueballs behave as stable particles that are free and noninteracting.

However, gravitational interactions of higher-spin particles are strongly constrained by causality [4-6]. In particular, a recent theorem on metastable higher-spin particles seems to suggest that glueballs or mesons of spin $J>2$ when coupled to gravity can be used to send signals outside of the lightcone [6]. In this paper, we discuss how this tension between causality and confining large $N$ gauge theories in $3+1$ dimensions can be resolved. We discuss two classes of resolutions: (I) gauge theory states might resolve this tension via a mixing with gravity or (II) new gravity sector states could remove the problem. Scenario (I) is certainly the more conservative resolution, as it does not involve any new states. However, for it to work a tower of spin-2 glueballs must remove causality violation due to pure graviton exchange between higher-spin glueballs. We believe this possibility merits further investigation, since the second scenario would have profound implications.

\section{CAUSALITY}

The theorem of [6] leverages recent advances constraining quantum field theories (QFTs) from causality [4,5,7-22]. The main idea parallels the philosophy of [23] which demonstrated that there are Wilsonian effective field theories without a consistent UV completion.

In QFT, the eikonal phase-shift $\delta(s, \vec{b})$, where $\vec{b}$ is the impact parameter, plays a crucial role since it is closely 


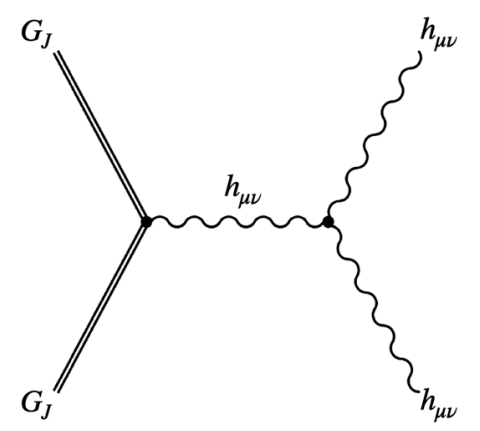

FIG. 1. This Feynman diagram in large $N$ QCD is inconsistent with causality for $J \geq 3$ unless it is accompanied by an infinite tower of additional higher-spin exchanges with unbounded spin. The same is true for mesons as well.

related to the Shapiro time-delay. In particular, causality requires that (i) $\delta(s, \vec{b})$ cannot grow faster than $s$, (ii) when $\delta(s, \vec{b})$ grows as $s$ it must be non-negative [9]. This imposes constraints on the scattering amplitude $\mathcal{A}(s, t)$ of the $2 \rightarrow 2$ scattering $G_{J} h_{\mu \nu} \rightarrow G_{J} h_{\mu \nu}$, where $h_{\mu \nu}$ is the graviton and $G_{J}$ is any glueball in large $N$ QCD with spin $J \geq 3$. It is well-known that only t-channel poles of $\mathcal{A}(s, t)$ contribute to the phase-shift. For weakly coupled gravity, $\mathcal{A}(s, t)$ must have a simple pole at $t=0$ which represents a graviton exchange. However, the contribution of the graviton pole to the phase-shift is inconsistent with the positivity condition $\delta(s, \vec{b}) \geq 0$ because of interference effects [4,6]. This implies that the Feynman diagram 1 for $J \geq 3$, by itself, is at odds with causality.

The bounds from [6], when applied to glueballs of large $N$ QCD, imply that this causality violation can only be avoided if the scattering amplitude $\mathcal{A}(s, t)$ contains other higher-spin $(J \geq 3)$ states in the t-channel with an upper bound on the mass $\Lambda_{\mathrm{HS}}$ of the lightest higher-spin state appearing in the t-channel [24]:

$$
\begin{aligned}
& \Lambda_{\mathrm{HS}} \lesssim \Lambda_{\mathrm{QCD}}\left(\frac{M_{\mathrm{pl}}}{N \Lambda_{\mathrm{QCD}}}\right)^{\gamma} \quad N \lesssim \frac{M_{\mathrm{pl}}}{\Lambda_{\mathrm{QCD}}}, \\
& \Lambda_{\mathrm{HS}} \lesssim \Lambda_{\mathrm{QCD}} \quad N \gtrsim \frac{M_{\mathrm{pl}}}{\Lambda_{\mathrm{QCD}}},
\end{aligned}
$$

where $0 \leq \gamma \leq \frac{1}{2}$. The fractional power of $M_{\mathrm{pl}} / N \Lambda_{\mathrm{QCD}}$ in Eq. (2) arises because of interference effects [6]. However, the exact value of $\gamma$ depends on form-factors and the knowledge of the spectrum and cannot be fixed by our argument. Moreover, causality necessarily requires that the Feynman diagram 1 must be accompanied by an infinite tower of higher-spin exchanges with unbounded spin $[4,9,25]$.

It is important to emphasize that the bound (2) has been derived at the leading order in $N$ under the only assumption that large $N$ confining gauge theories in $3+1$ dimensions, when coupled to gravity, do not violate causality at energies much below the scale $\Lambda_{\mathrm{HS}}$. Of course, the argument requires $N$ to be large enough so that $1 / N$ corrections to the analysis of [6] are suppressed [26]. Moreover, at large but finite $N$ higher-spin glueballs will likely decay to other QCD states. So we also require that there is at least one spin $>2$ glueball of mass $m$ and size $r$ with decay-time (in the center-of-mass frame) $t_{\text {decay }}>\frac{m^{2} r}{|\delta| s}$ in the setup of [6] to ensure that scattering processes occur before the glueball decays. From both these conditions, a lower bound on $N>$ $N_{\min }$ can be determine numerically above which the bound (2) holds, provided we know the spectrum and relevant formfactors. Nevertheless, $N_{\min }$ should not depend on the various scales in the problem, such as $\Lambda_{\mathrm{QCD}}, \Lambda_{\mathrm{HS}}$, or $M_{\mathrm{pl}}$ [27].

At this stage we conclude that there are two ways the conflict between large $N$ QCD and causality can be resolved:

(I) Glueball states-The scattering amplitude $\mathcal{A}(s, t)$ has additional glueball states appearing in the t-channel.

(II) Gravity states-The scattering amplitude $\mathcal{A}(s, t)$ develops new $\mathrm{t}$-channel poles that represent higher-spin states in the gravity sector.

Of course, the lightest higher-spin state appearing in the t-channel must obey the bound (2) in both cases. For the remainder of this paper, we will elaborate on these resolutions and argue that both have surprising implications.

\section{CAUSALITY RESTORATION BY GLUEBALL STATES}

At first sight, it seems counterintuitive to imagine that the gauge sector will remedy causality violations that are due to the graviton exchange. After all, we can replace the external gravitons by any other particle which is not in the QCD sector and the same problem persists. However, more careful consideration supports this possibility.

When large $N$ QCD is coupled to gravity, the scaling of matrix elements of glueballs with the graviton $h_{\mu \nu}$ are, in general, given by

$$
\left\langle G_{1} \cdots G_{n} h_{1} \cdots h_{m}\right\rangle \sim \frac{1}{N^{n-2} M_{\mathrm{pl}}^{m}} .
$$

Importantly, all matrix elements with a single glueball and one or more gravitons can be $N$-enhanced

$$
\left\langle G_{J} h_{\mu \nu}\right\rangle \sim \frac{N}{M_{\mathrm{pl}}}, \quad\left\langle G_{J} h_{\mu_{1} \nu_{1}} h_{\mu_{2} \nu_{2}}\right\rangle \sim \frac{N}{M_{\mathrm{pl}}^{2}}, \cdots
$$

unless they are tuned to be zero. The first matrix element represents a kinetic mixing between glueballs and the graviton. Of course, it is nonvanishing only when the graviton is off-shell and away from the soft-limit. Moreover, Lorentz invariance only allows mixing between spin-2 glueballs and the graviton [28]. The second matrix element corresponds to the decay of a glueball into two gravitons. These interactions can restore causality in the large $N$ limit.

The presence of an infinite set of $\left\langle G_{J} h_{\mu_{1} \nu_{1}} h_{\mu_{2} \nu_{2}}\right\rangle$ immediately implies that the scattering amplitude $\mathcal{A}(s, t)$, at the order $1 / M_{\mathrm{pl}}^{2}$, has additional glueball states appearing in the t-channel. Since glueballs have mass of parametric order 
$\Lambda_{\mathrm{QCD}}$, these glueball states can make $\mathcal{A}(s, t)$ consistent with the causality bound (2).

On the other hand, the kinetic mixing $m_{\mu_{1} \mu_{2}}^{\nu_{1} \nu_{2}}(p) \equiv$ $\left\langle G_{J=2}^{\nu_{1} \nu_{2}}(p) h_{\mu_{1} \mu_{2}}(-p)\right\rangle$ is already required to reproduce the 2-point function of the stress tensor [29]. In fact, one can derive a useful sum rule for such couplings

$$
\begin{aligned}
& \left\langle T_{\mu_{1} \mu_{2}}(p) T_{\mu_{3} \mu_{4}}(-p)\right\rangle \\
& \quad=\frac{M_{\mathrm{pl}}^{2}}{4} \sum_{J=2} \frac{m_{\mu_{1} \mu_{2}}^{\nu_{1} \nu_{2}}(p) m_{\mu_{3} \mu_{4}}^{\nu_{3} \nu_{4}}(p) \Pi_{\nu_{1} \nu_{2}, \nu_{3} \nu_{4}}(p)}{p^{2}+m_{J}^{2}}
\end{aligned}
$$

where the sum is over all spin-2 glueballs and $\Pi_{\nu_{1} \nu_{2}, \nu_{3} \nu_{4}}$ is the usual orthogonal projector for spin-2 exchanges. Note that the above sum rule implies that an infinite number of such mixing must be nonzero in order to produce a 2-point function of the stress tensor which is consistent with the asymptotic freedom [30]. Furthermore, in the presence of other non-QCD sectors, the kinetic mixing is also necessary in order to make large $N$ QCD causal without modifying the gravity sector. For example, the non-QCD sector could just be a free particle with only gravitational interactions. One can replace the external gravitons in Fig. 1 by any other particle $X$ (with or without spin) and extend the argument of [6] to conclude that the corresponding amplitude $G_{J} X \rightarrow G_{J} X$ must obey the same bound (2). However, now the causality violation can be avoided if there is a t-channel state below $\Lambda_{\mathrm{HS}}$ with spin $J \geq 2$ [31]. The kinetic mixing term (4) generates effective on-shell amplitudes $\left\langle X X G_{J=2}\right\rangle \sim N / M_{\mathrm{pl}}^{2}$ for all $X$. The effective interactions $\left\langle X X G_{J=2}\right\rangle$, in principle, can make the amplitude $G_{J} X \rightarrow$ $G_{J} X$ causal. Of course, causality is a more precise constraint, beyond the sum rule (5). Furthermore, the gravitonglueball kinetic mixing is also required in order to generate effective three-point amplitudes of glueballs

$$
\left\langle G_{J} G_{J^{\prime}}^{\prime} G_{J=2}\right\rangle \sim \frac{N}{M_{\mathrm{pl}}^{2}} .
$$

In contrast to the canonical large $N$ scaling (1), this contribution is enhanced at large $N$. This $N$-enhancement can, in principle, make the amplitude $G_{J} G_{J^{\prime}}^{\prime} \rightarrow G_{J} G_{J^{\prime}}^{\prime}$ consistent with causality in the presence of gravity.

One might think of criticizing this solution on the grounds that massive spin-2 interactions are known to be highly constrained [9,19,32-41]. Moreover, in certain situations, as discussed in [9], they can introduce additional causality violations. Thus, we do not know whether, and in what way, including a sum of spin-2 particles in the tchannel, can definitely save causality. We wish to emphasize that a better understanding of causality constraints when the graviton exchange is accompanied by a spin- 2 tower would have important implications for large $N$ QCD.

So, the causality violations caused by the graviton can be resolved by the glueball states only if the IR interactions (4) of glueballs take a specific form [42]. In this scenario, the coefficients of higher dimensional operators (4) in the IR effective theory are tightly constrained by causality. From the UV perspective, this IR "fine tuning" is unavoidable (but may be automatic from the UV Lagrangian). Whereas, from the IR perspective, it may appear that there has been a miraculous cancellation between the gravity sector and the QCD sector. Any low-energy terms that change these higher dimensional operators necessarily require new states in the gravity sector.

\section{A. Spectating particles and classical shockwaves}

The nontrivial mixing between the gauge sector and the gravity sector due to (4) cannot fix causality violation when spectating particles (with or without spin) are present in the theory. Because of the kinetic mixing, the meaning of "spectating" particles is subtle, so let us first give it a definite meaning. The scattering amplitude $\psi G_{J} \rightarrow \psi G_{J}$ of a spectating particle $\psi$ has t-channel poles only at the location of the graviton and other particles in the gravity sector (if any). This necessarily requires some fine-tuning because of the mixing (4). More physically, spectating particles are defined as particles that can create classical gravitational shockwave backgrounds, in the absence of additional gravity states. The preceding discussion implies that spectating particles and hence classical shockwaves are ruled out unless we introduce new states in the gravity sector.

\section{B. Free massless spin- $\frac{3}{2}$ particles are ruled out}

We now consider the scattering process $G_{J} X_{3 / 2} \rightarrow$ $G_{J} X_{3 / 2}$, where $X_{3 / 2}$ is a free massless spin- $\frac{3}{2}$ particle [43]. We can define a $2 \times 2$ phase-shift matrix $\delta_{ \pm, \pm}$, where + and - represent two helicities of the incoming and outgoing $X_{3 / 2}$. Causality requires $\delta$ to be positive semidefinite implying $\delta_{++}, \delta_{--} \geq 0$. An argument identical to the Weinberg-Witten theorem [44] ensures that the effective couplings $\left\langle X_{+} X_{+} G_{J=2}\right\rangle=\left\langle X_{-} X_{-} G_{J=2}\right\rangle=0$ because of angular momentum conservation, even when mixing terms (4) are present [45]. Moreover, other higher-spin $(J \geq 3)$ glueballs do not contribute as well at the leading order in $N$. This implies that glueball states cannot make the scattering process $G_{J} X_{3 / 2} \rightarrow G_{J} X_{3 / 2}$ causal. Thus, any theory of large $N$ QCD along with free massless spin- $\frac{3}{2}$ particles is inconsistent with causality unless there exist higher-spin states in the gravity sector obeying (2).

\section{The regime $n \gtrsim \frac{m_{\mathrm{pl}}}{\lambda_{\mathrm{qcd}}}$}

It follows from (4) that glueballs (and mesons), in the presence of gravity, become more and more unstable as we increase $N$ keeping $M_{\mathrm{pl}} / \Lambda_{\mathrm{QCD}}$ fixed. Furthermore, as $N \sim \frac{M_{\mathrm{pl}}}{\Lambda_{\mathrm{QCD}}}$, the mixing between the QCD and the gravity 
sectors become significantly large. In particular, consider the scattering process $h h \rightarrow h h$ which now receives contributions at the order $1 / M_{\mathrm{pl}}^{2}$ from higher-spin glueball exchanges because of interactions (4). If gravity is still weakly coupled and glueball states can be approximated by well-separated poles, the theorem of [25] implies that the resulting theory has a consistent S-matrix only if glueball states organize themselves into Regge trajectories that asymptotically coincide with the tree-level string theory spectrum, where the effective string scale is given by $\Lambda_{\mathrm{QCD}}$ [6] [46,47]. Thus, for $N \gtrsim \frac{M_{\mathrm{pl}}}{\Lambda_{\mathrm{QCD}}}$ the graviton amplitude, if weakly coupled, has a natural description in terms of strings. Moreover, in this regime, it is tempting to interpret glueballs as effective excitations of closed strings [48].

\section{CAUSALITY RESTORATION BY GRAVITY STATES}

We might instead resolve the causality problem caused by the graviton by modifying the gravity sector. In fact, there are some plausible-seeming situations where it is the only way to restore causality:

(i) Phenomenologically one may choose to add higher dimensional operators in the IR effective theory that modify some of the interactions (4).

(ii) Interactions (4) are present but do not actually solve the causality problem by itself.

(iii) Spectating particles (whose scattering amplitudes only have gravity-sector poles) are present.

(iv) A free massless spin- $\frac{3}{2}$ particle is present.

Again consider the scattering amplitude $\mathcal{A}(s, t)$ of the process $G_{J} h_{\mu \nu} \rightarrow G_{J} h_{\mu \nu}$. Now, the causality violation due to the graviton pole is fixed by new t-channel poles that should be regarded as higher-spin states in the gravity sector. Hence, metastable higher-spin glueballs (or mesons) in $3+1$ dimensions can couple to gravity while preserving causality if there exist higher-spin states in the gravitational sector well below the Planck scale $M_{\mathrm{pl}}$. Furthermore, inequalities (2) now impose a bound on the mass $\Lambda_{\mathrm{HS}}$ of the lightest higher-spin particle in the gravity sector.

The existence of these new gravitational states implies that for $N \gtrsim \frac{M_{\mathrm{pl}}}{\Lambda_{\mathrm{QCD}}}$ the QFT approximation breaks down at the scale of $\Lambda_{\mathrm{QCD}}$ and hence even in this scenario we do not have a QCD theory in the traditional sense [47].

\section{A. UV completion for $n \lesssim \frac{m_{\mathrm{pl}}}{\lambda_{\mathrm{qcd}}}$}

This scenario has profound implications that stem from the fact that theories with higher-spin exchanges are highly constrained by S-matrix consistency conditions [9,25]. First of all, any four-point amplitude with a finite number of higher-spin exchanges is inconsistent with causality since a spin- $J$ exchange produces a phase shift $\delta \sim s^{J-1}$. Thus, in this scenario, the gravity sector necessarily

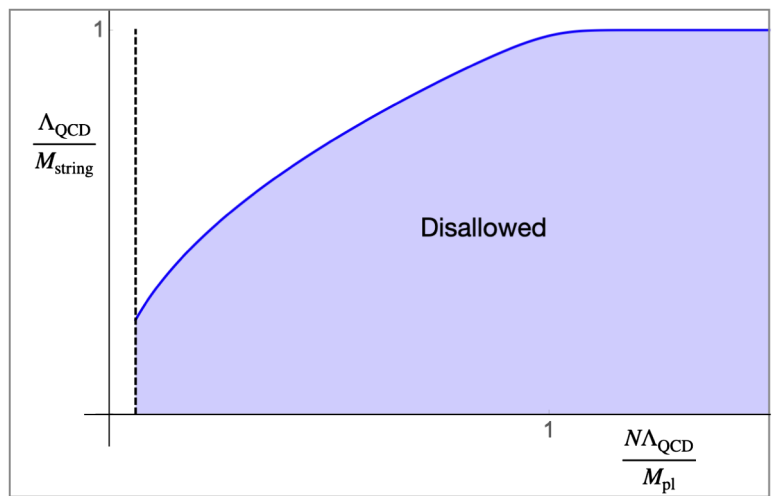

FIG. 2. A schematic exclusion plot for the string scale $M_{\text {string }} \approx$ $\Lambda_{\mathrm{HS}}$ as a function of $N$ when gravity states are restoring causality. The solid blue line represents the bound (2) for $\gamma=1 / 2$, where the shaded region is ruled out by causality. For $\gamma<1 / 2$, the bound asymptotes to 1 at a faster rate. The dashed black line corresponds to an (unknown) large but finite value of $N$ above which our bounds hold.

contains the graviton and an infinite tower of higher-spin particles above $\Lambda_{\mathrm{HS}}$ with unbounded spin [64]. Furthermore, we assume that the gravity sector of the underlying UV complete theory is weakly coupled and has a healthy thermodynamic limit. This implies that the gravity spectrum does not have an accumulation point and the leading gravitational scattering amplitude is a meromorphic function with simple poles obeying unitarity, causality, and crossing symmetry. Now invoking the theorems of $[6,25]$ we conclude that the full gravity sector must contain infinite towers of Regge trajectories that are asymptotically parallel, linear, and equispaced. In particular, the gravitational scattering amplitude in the regime $t, s \rightarrow \infty$ (such that all intermediate scales decouple) must coincide with the tree-level four-point amplitude of fundamental closed strings $[6,25,65]$

$$
\lim _{s, t \gg 1} A_{\text {gravity }}(s, t)=A_{0} e^{-\frac{\alpha^{\prime}}{2}(u \ln u+s \ln s+t \ln t)},
$$

where the Regge slope is $\alpha^{\prime} \approx \frac{1}{\Lambda_{\mathrm{HS}}^{2}}$. The above amplitude has the feature that the inelastic part even for large impact parameter is nonzero and consistent with the production of long strings. Thus, in this scenario $\Lambda_{\mathrm{HS}}$ should be identified as the string scale $M_{\text {string }} \approx \Lambda_{\mathrm{HS}}$. This is perfectly consistent with the fact that infinite towers of higher-spin states in string theory have a well-behaved S-matrix which respects asymptotic causality [66-71].

Therefore, when gravity states restore causality of a confining large $N$ gauge theory, any weakly coupled UV completion of the resulting theory must have a gravity sector with many of the properties of fundamental strings [72]. The bound (2), in the present context, has a natural interpretation as a bound on the string scale. The claimed bound on the string scale, as summarized in Fig. 2, appears 
quite surprising. For example, even a conservative estimate of (2) implies a parametric bound

$$
M_{\text {string }} \lesssim \sqrt{M_{\mathrm{pl}} \Lambda_{\mathrm{QCD}} / N} \quad \text { when } N \lesssim \frac{M_{\mathrm{pl}}}{\Lambda_{\mathrm{QCD}}}
$$

but large $N>N_{\min }$ [73], irrespective of the details of the QCD sector. On the other hand, $M_{\text {string }}$ must be at or below $\Lambda_{\mathrm{QCD}}$ for $N \gtrsim \frac{M_{\mathrm{pl}}}{\Lambda_{\mathrm{QCD}}}$, where scenarios (I) and (II) become practically indistinguishable.

The above predictions are rather surprising, so one may try to find counterexamples to conclusively rule out the scenario (II). At first sight, the bound (8) seems to be in tension with the heterotic string theory. For example, one can start with heterotic strings in $10 \mathrm{~d}$ and study compactification to $4 \mathrm{~d}$ Minkowski space with $\mathcal{N}=2$ supersymmetry [74]. In this construction, $\Lambda_{\mathrm{QCD}} / M_{\text {string }}$ is nonperturbatively small in the string coupling and hence appears to violate (8). However, these types of constructions can only provide a confining gauge theory coupled to gravity with $N$ up to order 10 . There are other string constructions with similar features that can lead to slightly higher $N$, see [75] for example. It is not actually a contradiction because our bound is applicable only above some large but finite $N>N_{\min }$, where the $N_{\min }$ is theory dependent but should be independent of $M_{\mathrm{pl}}, \Lambda_{\mathrm{QCD}}$ or $\Lambda_{\mathrm{HS}}$. Indeed, we are not aware of any construction where $N$ is arbitrarily large providing a concrete counterexample.

At this stage, one may propose a natural question: what is the largest gauge group for a confining gauge theory coupled to gravity obtained from an explicit string construction?

\section{CONCLUSIONS}

Metastable higher-spin glueballs (and mesons) in four spacetime dimensions are only consistent with causality when other higher-spin states contribute to gravitational scattering amplitudes $[76,77]$. We have argued for an upper bound on the mass $\Lambda_{\mathrm{HS}}$ of the lightest higher-spin state required for the preservation of causality. These higher-spin states can come from the glueball sector because of a nontrivial mixing between QCD and gravity. This rules out the existence of spectating particles that create classical shockwaves and massless free spin- $\frac{3}{2}$ particles. Causality can also be restored by a stringy gravity sector in which case we obtain a surprisingly strong bound on the string scale. A unifying feature of both these resolutions is that for large $N \gtrsim \frac{M_{\mathrm{pl}}}{\Lambda_{\mathrm{QCD}}}$ the gravity sector of the resulting theory, if weakly coupled, only has a stringy description with the string scale at or below $\Lambda_{\mathrm{QCD}}$.

\section{ACKNOWLEDGMENTS}

It is our pleasure to thank Nima Afkhami-Jeddi, Simon Caron-Huot, Liam Fitzpatrick, Shamit Kachru, David Kaplan, Ami Katz, Juan Maldacena, and Amirhossein Tajdini for several helpful discussions. We would also like to thank Simon Caron-Huot and Juan Maldacena for comments on a draft. We were supported in part by the Simons Collaboration Grant on the Non-Perturbative Bootstrap. J. K. was supported in part by NSF Grant No. PHY-1454083.
[1] G. 't Hooft, A planar diagram theory for strong interactions, Nucl. Phys. B72, 461 (1974).

[2] G. 't Hooft, A two-dimensional model for mesons, Nucl. Phys. B75, 461 (1974).

[3] E. Witten, Baryons in the 1/n expansion, Nucl. Phys. B160, 57 (1979).

[4] N. Afkhami-Jeddi, S. Kundu, and A. Tajdini, A bound on massive higher-spin particles, J. High Energy Phys. 04 (2019) 056.

[5] J. Kaplan and S. Kundu, A species or weak-gravity bound for large $N$ gauge theories coupled to gravity, J. High Energy Phys. 11 (2019) 142.

[6] J. Kaplan and S. Kundu, Closed strings and weak gravity from higher-spin causality, J. High Energy Phys. 02 (2021) 145.

[7] D. M. Hofman and J. Maldacena, Conformal collider physics: Energy and charge correlations, J. High Energy Phys. 05 (2008) 012.
[8] D. M. Hofman, Higher derivative gravity, causality and positivity of energy in a UV complete QFT, Nucl. Phys. B823, 174 (2009).

[9] X. O. Camanho, J. D. Edelstein, J. Maldacena, and A. Zhiboedov, Causality constraints on corrections to the graviton three-point coupling, J. High Energy Phys. 02 (2016) 020.

[10] T. Hartman, S. Jain, and S. Kundu, Causality constraints in conformal field theory, J. High Energy Phys. 05 (2016) 099.

[11] T. Hartman, S. Jain, and S. Kundu, A new spin on causality constraints, J. High Energy Phys. 10 (2016) 141.

[12] J. D. Edelstein, G. Giribet, C. Gomez, E. Kilicarslan, M. Leoni, and B. Tekin, Causality in 3D massive gravity theories, Phys. Rev. D 95, 104016 (2017).

[13] D. M. Hofman, D. Li, D. Meltzer, D. Poland, and F. RejonBarrera, A proof of the conformal collider bounds, J. High Energy Phys. 06 (2016) 111. 
[14] T. Hartman, S. Kundu, and A. Tajdini, Averaged null energy condition from causality, J. High Energy Phys. 07 (2017) 066.

[15] N. Afkhami-Jeddi, T. Hartman, S. Kundu, and A. Tajdini, Einstein gravity 3-point functions from conformal field theory, J. High Energy Phys. 12 (2017) 049.

[16] X. O. Camanho, G. Lucena Gomez, and R. Rahman, Causality constraints on massive gravity, Phys. Rev. D 96, 084007 (2017).

[17] N. Afkhami-Jeddi, T. Hartman, S. Kundu, and A. Tajdini, Shockwaves from the operator product expansion, J. High Energy Phys. 03 (2019) 201.

[18] K. Hinterbichler, A. Joyce, and R. A. Rosen, Eikonal scattering and asymptotic superluminality of massless higher-spin fields, Phys. Rev. D 97, 125019 (2018).

[19] J. Bonifacio, K. Hinterbichler, A. Joyce, and R. A. Rosen, Massive and massless spin-2 scattering and asymptotic superluminality, J. High Energy Phys. 06 (2018) 075.

[20] N. Afkhami-Jeddi, S. Kundu, and A. Tajdini, A conformal collider for holographic CFTs, J. High Energy Phys. 10 (2018) 156.

[21] T. A. Chowdhury, R. Rahman, and Z. A. Sabuj, Gravitational properties of the proca field, Nucl. Phys. B936, 364 (2018).

[22] S. Kundu, A generalized Nachtmann theorem in CFT, J. High Energy Phys. 11 (2020) 138.

[23] A. Adams, N. Arkani-Hamed, S. Dubovsky, A. Nicolis, and R. Rattazzi, Causality, analyticity and an IR obstruction to UV completion, J. High Energy Phys. 10 (2006) 014.

[24] The same conclusion holds even for mesons which leads to a weaker bound. The bound for mesons can be obtained from (2) by replacing $N$ by $\sqrt{N}$.

[25] S. Caron-Huot, Z. Komargodski, A. Sever, and A. Zhiboedov, Strings from massive higher-spins: The asymptotic uniqueness of the Veneziano amplitude, J. High Energy Phys. 10 (2017) 026.

[26] The leading $1 / N$ correction comes from the phase-shift associated with the mixed amplitude $G_{J} G_{J} \rightarrow h_{\mu \nu} h_{\mu \nu}$ which led to the interference bound of Ref. [6].

[27] It is also possible that width of glueball states, for large but finite $N$, increases significantly as one moves up the spectrum. So, these states may not be approximated by well-separated poles. However, this effect does not change the results of this article (unless stated explicitly) as long as glueballs states are well-separated from the graviton pole.

[28] The only nonvanishing kinetic mixing term that can be written has the structure $h_{\mu \nu} \square^{k} G_{J=2}^{\mu \nu}$.

[29] We thank J. Maldacena for pointing this out to us.

[30] The mixing between gravitons and spin-2 mesons/glueballs is simply a spin-2 analogue of the mixing of photons with $\rho$ mesons that has been familiar in QCD for several decades.

[31] For external gravitons, as explained in Ref. [9], even an infinite tower of massive spin-2 exchanges cannot solve the problem. This is true for any external massless particle with spin $\geq \frac{3}{2}$.

[32] C. Aragone and S. Deser, Consistency problems of spin-2 gravity coupling, Nuovo Cimento Soc. Ital. Fis. 57B (1980) 33.
[33] I. Buchbinder, D. Gitman, V. Krykhtin, and V. Pershin, Equations of motion for massive spin-2 field coupled to gravity, Nucl. Phys. B584, 615 (2000).

[34] I. Buchbinder, V. Krykhtin, and V. Pershin, On consistent equations for massive spin two field coupled to gravity in string theory, Phys. Lett. B 466, 216 (1999).

[35] I. Buchbinder, D. Gitman, and V. Pershin, Causality of massive spin-2 field in external gravity, Phys. Lett. B 492, 161 (2000).

[36] Y. Zinoviev, On massive spin 2 interactions, Nucl. Phys. B770, 83 (2007).

[37] N. Arkani-Hamed, T.-C. Huang, and Y.-t. Huang, Scattering amplitudes for all masses and spins, arXiv:1709.04891.

[38] J. J. Bonifacio, Aspects of massive spin-2 effective field theories, Ph.D. thesis, Oxford U., 2017.

[39] J. Bonifacio and K. Hinterbichler, Universal bound on the strong coupling scale of a gravitationally coupled massive spin-2 particle, Phys. Rev. D 98, 085006 (2018).

[40] D. Klaewer, D. Lust, and E. Palti, A spin-2 conjecture on the swampland, Fortschr. Phys. 67, 1800102 (2019).

[41] C. De Rham, L. Heisenberg, and A. J. Tolley, Spin-2 fields and the weak gravity conjecture, Phys. Rev. D 100, 104033 (2019).

[42] It is also possible that interactions (4), however fine tuned, cannot actually solve the problem by itself.

[43] In a gravitational theory, free particles should be defined carefully. To be precise, by free particle we mean that the spin- $\frac{3}{2}$ particle interacts (not necessarily minimally) only with gravitons. Of course, because of the mixing (4) interactions like $\left\langle X_{-} X_{+} G_{J=2}\right\rangle$ are also nonzero. However, all other interactions $\left\langle X_{3 / 2} X_{3 / 2} G_{J \neq 2}\right\rangle$ are still absent at the leading order in $N$.

[44] S. Weinberg and E. Witten, Limits on massless particles, Phys. Lett. 96B, 59 (1980).

[45] The argument is essentially the same as that in Ref. [9] for the decay of a massive spin-2 particle into two gravitons.

[46] Strictly speaking, the theorem of Ref. [25] is applicable only for external scalars. However, it is expected that the same argument holds even for spinning external states. This is supported by the fact that high energy string amplitudes are independent of the exact quantum numbers of external states.

[47] In Ref. [5], a parametric bound on $N$ was derived under some additional assumptions. One of the assumptions was that the scale of new physics, such as $\Lambda_{\mathrm{HS}}$, is parametrically higher than $\Lambda_{\mathrm{QCD}}$. From the bound (2) it is clear that any parametric separation between $\Lambda_{\mathrm{HS}}$ and $\Lambda_{\mathrm{QCD}}$ necessarily requires $N \lesssim \frac{M_{\mathrm{pl}}}{\Lambda_{\mathrm{QCD}}}$ which agrees with the bound of Ref. [5].

[48] The connection between glueballs and closed strings has a long and interesting history. For example see Refs. [49-63].

[49] G. Bhanot and C. Rebbi, SU(2) String tension, glueball mass and interquark potential by Monte Carlo computations, Nucl. Phys. B180, 469 (1981).

[50] A. J. Niemi, Are glueballs knotted closed strings? arXiv: hep-th/0312133.

[51] G. Sharov, Closed string with masses in models of baryons and glueballs, arXiv:0712.4052.

[52] L. Solovev, Glueballs in the string quark model, Theor. Math. Phys. 126, 203 (2001). 
[53] S. Talalov, The glueball Regge trajectory from the string inspired theory, arXiv:hep-ph/0101028.

[54] F. J. Llanes-Estrada, S. R. Cotanch, P. J. de A. Bicudo, J.E. F. Ribeiro, and A.P. Szczepaniak, QCD glueball Regge trajectories and the Pomeron, Nucl. Phys. A710, 45 (2002).

[55] A. P. Szczepaniak and E. S. Swanson, The low lying glueball spectrum, Phys. Lett. B 577, 61 (2003).

[56] J. Pons, J. Russo, and P. Talavera, Semiclassical string spectrum in a string model dual to large N QCD, Nucl. Phys. B700, 71 (2004).

[57] E. Abreu and P. Bicudo, Glueball and hybrid mass and decay with string tension below Casimir scaling, J. Phys. G 34, 195 (2007).

[58] F. Brau and C. Semay, Semirelativistic potential model for glueball states, Phys. Rev. D 70, 014017 (2004).

[59] V. Mathieu, C. Semay, and F. Brau, Casimir scaling, glueballs and hybrid gluelumps, Eur. Phys. J. A 27, 225 (2006).

[60] Y. Simonov, Glueballs, gluerings and gluestars in the $\mathrm{d}=$ $2+1$ SU(N) gauge theory, Phys. At. Nucl. 70, 44 (2007).

[61] V. Mathieu, C. Semay, and B. Silvestre-Brac, Semirelativistic potential model for low-lying three-gluon glueballs, Phys. Rev. D 74, 054002 (2006).

[62] H. Boschi-Filho and N. R. Braga, Gauge/string duality and scalar glueball mass ratios, J. High Energy Phys. 05 (2003) 009.

[63] J. Sonnenschein and D. Weissman, Glueballs as rotating folded closed strings, J. High Energy Phys. 12 (2015) 011.

[64] There is a small loophole that we must close. In certain situation [for example the situation (iii)] causality, in principle, can be restored by one or more spin-2 states. However, since these states are now in the gravity sector, they will appear in the 4-graviton scattering as well. Spin-2 exchanges lead to additional causality violations [9] in the 4-graviton scattering unless they are accompanied by other higher-spin $(J \geq 3)$ states in the gravity sector.

[65] D. J. Gross and P. F. Mende, The high-energy behavior of string scattering amplitudes, Phys. Lett. B 197, 129 (1987).
[66] G. D’Appollonio, P. Di Vecchia, R. Russo, and G. Veneziano, Regge behavior saves string theory from causality violations, J. High Energy Phys. 05 (2015) 144.

[67] D. Amati, M. Ciafaloni, and G. Veneziano, Superstring collisions at Planckian energies, Phys. Lett. B 197, 81 (1987).

[68] D. Amati, M. Ciafaloni, and G. Veneziano, Classical and quantum gravity effects from Planckian energy superstring collisions, Int. J. Mod. Phys. A 03, 1615 (1988).

[69] D. Amati, M. Ciafaloni, and G. Veneziano, Can space-time be probed below the string size?, Phys. Lett. B 216, 41 (1989).

[70] D. Amati, M. Ciafaloni, and G. Veneziano, Planckian scattering beyond the semiclassical approximation, Phys. Lett. B 289, 87 (1992).

[71] D. Amati, M. Ciafaloni, and G. Veneziano, Effective action and all order gravitational eikonal at Planckian energies, Nucl. Phys. B403, 707 (1993).

[72] Let us emphasize that this conclusion is valid only when the UV completion is weakly coupled. In fact, there are M-theory compactifications on manifolds with singularities that give rise to confining large $N$ gauge theories which have strongly coupled UV completion.

[73] Note that the lower cut-off $N_{\min }$ is defined after Eq. (2).

[74] S. Kachru and C. Vafa, Exact results for $N=2$ compactifications of heterotic strings, Nucl. Phys. B450, 69 (1995).

[75] S. Kachru, A. Klemm, W. Lerche, P. Mayr, and C. Vafa, Nonperturbative results on the point particle limit of $N=2$ heterotic string compactifications, Nucl. Phys. B459, 537 (1996).

[76] To be clear, if large $N$ confining theories somehow do not contain higher spin glueballs in their spectra, none of our arguments would apply.

[77] It is possible that a combination of scenario (I) and (II) restores causality in such a way that the bound (8) can be relaxed. For example, before we hit the scale on the right hand side of (8), there may be a transition from scenario (II) to scenario (I). In this case, the bound (8) on the string scale is no longer reliable. 